\title{
Modification of Magerl's technique for the placement of translaminar facet screws in transforaminal lumbar interbody fusion: a technical note and comparative outcome analysis
}

\author{
Daoliang Xu \\ second affiliated hospital of Wenzhou Medical University \\ Haimin Jin \\ Wenzhou Medical University Second Affiliated Hospital \\ Jiaoxiang Chen \\ Wenzhou Medical University Second Affiliated Hospital \\ Xiangyang Wang ( $\sim$ knightman@yeah.net )
}

\section{Technical note}

Keywords: Modified technique, Translaminar facet screws, Transforaminal lumbar interbody fusion

Posted Date: April 29th, 2020

DOI: https://doi.org/10.21203/rs.3.rs-22186/v1

License: (c) (i) This work is licensed under a Creative Commons Attribution 4.0 International License.

Read Full License 


\section{Abstract \\ Background}

To describe and illustrate a safe and effective technique for the placement of translaminar facet screws (TLFS) in transforaminal lumbar interbody fusion (TLIF).

\section{Methods}

Forty-two patients with single-level lumbar diseases were divided into two groups randomly. 21 patients were treated by traditional TLIF using bilateral pedicle screws fixation (BPS) while the other patients underwent insertion of a unilateral pedicle screw (UPS)and contralateral TLFS using our modified technique. In this technique, a small unicortical "hole" was formed adjacent to the contralateral facet joint to ensure that insertion of the screw could be directly visualized through the hole to prevent violation of the spinal canal. The ODI, JOA, VAPS questionnaire, the mean operation time, mean operative blood loss, length of stay and postoperative complications were collected for analysis.

\section{Results}

There is no significant difference between the BPS and UPS + TLFS group in the preoperative and postoperative ODI, JOA or VAPS at each follow- up visit, while the UPS + TLFS group using our modified technique significantly reduced the mean operation time, the mean estimated blood loss and the length of stay. These results demonstrated this modified technique to be safe and effective in TLIF.

\section{Conclusions}

In contrast to conventional TLIF, our modified technique for placing TLFS in TLIF can reduce soft tissue injuries, reduce the operation risk of violation of the spinal canal and the expenses, minimize radiation exposure, and shorten the length of the operation without a concurrent reduction in clinical efficacy.

\section{Introduction}

Transforaminal lumbar interbody fusion (TLIF) with bilateral pedicle screws (BPS), an important lumbar fixation method initially popularized in 1982 by Harms and Rolinger [1], has been widely utilized in the treatment of spinal instability and degenerative disc disease. It has been shown to be an effective and safe technique that can provide rigid fixation and increase the rate of fusion [2,3]. However, to implant the pedicle screw, extensive areas of bilateral paravertebral tissue must be stripped, and this may cause an increase in postoperative pain, prolonged recovery time, and impaired spinal function. These disadvantages led surgeons to pursue a less invasive posterior fixation technique, and a unilateral pedicle screw (UPS) was thus proposed for lumbar fixation and fusion. Although the UPS is capable of achieving 
a clinical fusion rate equivalent to that of the BPS [4], concerns about the adequacy of the UPS for stabilization, owing to its inherent construct asymmetry, have been raised [5]. Consequently, a hybrid TLIF composed of a UPS plus a single contralateral translaminar facet screw (TLFS) was proposed [6], which would significantly increase the degree of stabilization. Some studies have demonstrated good clinical results using this hybrid technique $[7,8]$. This method was able to maintain instant and long-term equivalent biomechanical ability, while being less invasive than the traditional BPS $[5,9]$.

However, the placement of the TLFS is also prone to complications including partial dorsal laminar breach and violation of the spinal canal. Moreover, it is a complicated procedure that should be performed with the aid of a customized guide device or under X-ray guidance. To address these issues, we have adjusted the hybrid technique to create a new minimally invasive technique for the placement of TLFS in TLIF. In our new technique, we slightly modified Magerl's method by creating a small unicortical "hole" adjacent to the facet joint at the contralateral dorsal lamina to ensure that the screw is directly in line with the lamina and has not violated the spinal canal under direct vision; thus, it can safely traverse the facet joints and come to rest at the base of the opposite transverse process of the lower vertebra. This study is to confirm the clinical curative effectiveness and other advantages of our modified technique, compared to conventional TLIF.

\section{Materials And Methods}

\section{Patients}

All procedures in this study were approved by The Affiliated Hospital and Yuying Children's Hospital of Wenzhou Medical University Research Ethics Committee. All patients gave informed written consent before inclusion in the study. Intact spinous process, lamina and an intact anterior column that is able to resist compressive forces are prerequisites for considering the use of TLFS [10,11], which was identically considered as our inclusion \& exclusion criteria. Between March 2016 and December 2016, 42 patients with single-level and unilateral symptomatic lumbar diseases were divided into two groups randomly. Patients in one group underwent insertion of a UPS supplemented with a single contralateral TLFS via our technique, while the other group was treated by traditional TLIF using BPS. All patients underwent preoperative magnetic resonance imaging, computed tomography (CT), and lumbar spine X-rays for the purposes of preoperative evaluation and planning. Postoperatively, the dynamic X-rays and CT were obtained to assess the biomechanical stability and fusion.

\section{Surgical Technique}

Intraoperatively, the neurophysiological monitoring of lumbar spinal nerve roots was used to ensure no neurological deficit. Under general anesthesia, patients were placed into the prone position with the hips at maximum extension conducive to maintaining lumbar lordosis. Then, a $4 \mathrm{~cm}$ median incision was made. Following satisfactory exposure of the posterior lumbar spine on the side of the most serious 
lesion or nerve root symptoms through subperiosteal approach, the pedicle screw was initially inserted on the ipsilateral side. In the BPS group, the contralateral pedicle screw was inserted similarly to the prior side from the same incision. Subsequent decompression of the neural structures, discectomy, end-plate decortication, and fusion were performed in the conventional manner.

In the UPS + TLFS group, further decompression would be inevitable in cases where some patients experienced preoperative nerve root compression symptoms on the contralateral side. Then, along with the spinous process, the contralateral partial paravertebral tissues were dissected to expose the lamina that adjacent to the facet joint (approximately $1.0-1.5 \mathrm{~cm}$ lateral to the midline), to create the unicortical "hole". A diamond drill was used to form a small cortical window in the middle of the exposed lamina, and cancellous bone was removed with rasps and curettes while preserving the medial lamina. After creation of a unicortical "hole," the next step was to insert the TLFS. The entry point as the ipsilateral anatomical marker was identified as the junction of the lamina and the base of the upper $1 / 3$ spinous process [12], consistent with previous study. Using a hand drill, the contralateral lamina was carefully drilled toward the facet joint. According to the previous literature [13], the drill aligned along the angle of the exposed contralateral laminar surface, aiming for the unicortical "hole" of the exposed lamina. The drill was visually confirmed to be drilling through the "hole" and into the cancellous laminar bone to prevent the drill from violating the spinal canal. Then, a blunt ball-tipped probe was inserted to ensure no cortical breakthrough into the spinal canal, and the length of the trajectory was palpated. Finally, a cortical bone screw of an appropriate diameter, was then inserted against the dorsal cortices of the lamina (Fig. 1). After hemostasis and rinsing with normal saline, a drain was inserted as required and the incision was sutured in layers.

\section{Clinical Assessment}

The preoperative and postoperative ODI, JOA and VAPS questionnaire were completed. The operative time and intraoperative blood loss were recorded. The postoperative mean follow-up was $31.2 \pm$ 8.7 months (range 6-42 months) in BPS group and 28.5 \pm 2.9 (range 22-33 months) in the UPS + TLFS group, respectively. Postoperative AP, lateral and dynamic X-rays, and three-dimensional CT scans were obtained to evaluate the imaging outcomes and biomechanical stability at the intervals of 6 weeks, 3, 6 months, 1, 2 and 3 years. The criteria for radiologic bony fusion according to the previous study [14].

\section{Statistical analysis}

The data was analyzed by the Wilcoxon Ranksum and T test were using the SPSS 15.0 (SPSS, Chicago, IL, USA). A p value less than 0.05 was considered statistically significant.

\section{Results}

Compared with the BPS group $(210.2 \pm 48.3 \mathrm{~min})$, the mean operation time in UPS + TLFS group (126.3 \pm $28.8 \mathrm{~min}$ ) significantly decreased. Likewise, both the mean estimated intraoperative blood loss and the 
length of stay UPS + TLFS group had a significant decrease, $240.3 \pm 72.7$ versus $428.5 \pm 79.6 \mathrm{ml}, 3.8 \pm 1.2$ versus $4.8 \pm 1.4$ days, respectively. The preoperative and postoperative ODI, JOA and VAPS scores exhibited no significant differences between the BPS and UPS + TLFS group (Table 1). No immediate intraoperative or early postoperative complications occurred in any of the 42 patients. Only one case of superficial infection was found and another patient was lost to follow-up in the BPS group at 6 postoperative months. Postoperative dynamic X-rays and CT scans showed that the postoperative stability and fusion rate in both group (95.2\% in UPS + TLFS versus $90.5 \%$ in BPS group) were excellent, and the position of the screws in our modified technique were satisfactory in all patients without violation of the spinal canal (Fig. 2).

Table 1

Demographic data and the results of patients

\begin{tabular}{|c|c|c|c|}
\hline & UPS + TLFS & BPS & p value \\
\hline ODI (Preoperative) & $28.9 \pm 6.0$ & $30.4 \pm 6.3$ & 0.4 \\
\hline ODI (3 months) & $3.6 \pm 1.3$ & $3.4 \pm 1.3$ & 0.6 \\
\hline JOA (Preoperative) & $11.4 \pm 1.8$ & $10.7 \pm 1.6$ & 0.2 \\
\hline JOA (3 months) & $23.3 \pm 3.0$ & $22.8 \pm 2.8$ & 0.5 \\
\hline VAPS (Preoperative) & $6.7 \pm 1.7$ & $7.1 \pm 18$ & 0.5 \\
\hline VAPS (3 months) & $1.5 \pm 0.6$ & $1.8 \pm 0.9$ & 0.2 \\
\hline Operative time(min) & $126.3 \pm 28.8$ & $210.2 \pm 48.3$ & $\varangle 0.01$ \\
\hline Estimated blood loss(ml) & $240.3 \pm 72.7$ & $428.5 \pm 79.6$ & $₫ 0.01$ \\
\hline Length of stay(d) & $3.8 \pm 1.2$ & $4.8 \pm 1.4$ & $₫ 0.05$ \\
\hline Mean follow-up & $30.5 \pm 8.7$ & $32.0 \pm 4.9$ & 0.5 \\
\hline Fusion at last follow-up & $20(95.2 \%)$ & $19(90.5 \%)$ & \\
\hline Complications & $0(0 \%)$ & $1(5 \%)$ & \\
\hline
\end{tabular}

\section{Discussion}

With the development of surgical technology, bilateral nerve roots can be decompressed via the single transforaminal approach [8]. With this method, the hybrid technique using a combination of a UPS plus a single contralateral TLFS in TLIF, pioneered by Jang et al. in 2005 [6], has gained increasing popularity due to diminished soft tissue injuries, reduced estimated blood loss, lower operative costs, and reduced potential risk of neurological injury $[6,8,15]$, compared with the standard BPS method. Recent biomechanical data suggest that the new fixation system provides the same degree of stability and supports the same amount of stiffness in all directions, such as flexion-extension, lateral bending, and axial rotation, compared with the BPS method $[9,16-18]$. Moreover, some clinical assessments 
demonstrated that although there was no significant difference between the two methods in terms of clinical outcome, fusion rate, or complication rate, the operative time, blood loss, and cost were significantly reduced in the hybrid method $[7,8]$.

However, the hybrid technique is technically more challenging and has been associated with neurological injuries. During insertion of the TLFS, which was devised by Magerl [19] as a less invasive alternative for posterior pedicle screw stabilization, the incidence of intraoperative complications including partial dorsal laminar breach or penetration of the screw into the spinal canal could not be completely avoided [20,21]. In addition, increased exposure to intraoperative radiation should not be ignored. To increase the safety and accuracy of TLFS placement, Grob and Humke [10] described a prototype device that they invented to percutaneously insert the TLFS as a supplementary posterior fixation method to anterior lumbar interbody fusion (ALIF). However, their study lacked technical details and clinical outcomes. Jang et al. [22] introduced a guide device for the percutaneous placement of the TLFS after ALIF. Unfortunately, their device is not commercially available and, thus, their technique cannot yet be widely used. Shim et al. [23] reported on their experience with a fluoroscopy-assisted percutaneous TLFS fixation technique without the use of a guide device. Although this technique allows the surgeon to obtain simultaneous intraoperative multilevel visualization of the proposed screw trajectory under fluoroscopic guidance, $10 \%$ of the screws violated the lamina wall, with $15 \%$ of the screws found to be in an imperfect position. Recently, some studies have demonstrated that the use of CT, or a combination of CT and fluoroscopic guidance, can dramatically reduce the difficulty in implanting the TLFS by providing both threedimensional landmarks and real-time imaging [24, 25]. However, these techniques may increase the exposure to radiation and the operation time, and the potential for inaccurate screw placement cannot be completely avoided.

Besides the auxiliary methods, a detailed knowledge of facet anatomy and corresponding radiographic criteria is required for the safe placement of a contralateral TLFS. Lu et al. [13] conducted an anatomic study in 30 dried lumbar spines to measure the screw path length, caudal and lateral angles, and superior and inferior lamina border thicknesses from L1 to L5 for insertion of the TLFS. In the human cadaveric study by Phillips et al. [26] the radiographic data suggested that the radiographic views identified to achieve proper placement of the TLFS were a true lateral, AP, $45^{\circ}$ oblique, and AP view with the X-ray beam at a $30^{\circ}$ to $45^{\circ}$ cephalad angle ("spinal outlet" view). These studies provide the relevant data for use of the TLFS and may greatly increase the safety of screw placement. In our study, most patients obtained bicortical purchase. On the one hand, based on the preoperative measurement of 3-dimensional image reconstruction of the CT data of patients and the intraoperative length of probe, the length of TLFS was ensure to be long enough to traverse the facet joints. On the other hand, considering that Asians are smaller in size than Westerner, the TLFS used in our study was $4.0 \mathrm{~mm}$ in diameter, which had been proved to be effective [8]. (Magerl inserted a $4.5 \mathrm{~mm}$ cortical screw for placing TLFS.) It is vital to ensure that the diameter of screw was no greater than the thickness of inferior border of the lamina, to prevent the lamina from occurring the bicortical purchase. 
To ensure the safe placement of a contralateral TLFS in the hybrid technique, we slightly modified Magerl's technique by adding a small unicortical "hole" adjacent to the facet joint at the contralateral dorsal lamina. Direct visualization of the insertion of the screw against the dorsal cortices of the lamina provides assurance, without the need for fluoroscopy, that the TLFS can be correctly located within the lamina and not entered the spinal canal. Compared with the conventional TLIF technique, our modified technique appears to be technically simple and safe, less invasive, and less expensive. We encountered no screw loosening or breakage and no neurologic injuries, and we attribute the absence of intraoperative complications to the fact that the insertion of the screws was technically easy under direct vision.

Clinically, compared to TLIF, the total operating time and estimated blood loss of the patients treated with our modified technique were reduced, which indicates that the new technique could help minimize the degree of surgical invasion and reduce the length of hospital stay. Moreover, the fusion rate (90.5\%) at last follow-up and the position of the screws were satisfactory according to the postoperative CT. Biomechanical stability was ensured by postoperative dynamic X-rays. These results demonstrate that our technique has clinical efficacy and safety, and this appears to be consistent with some of the more recent studies [6-8].

In summary, our modified technique results in significant improvements in both clinical safety and efficacy during the placement of TLFS. Moreover, it reduces the operation time and blood loss and incurs a lower operative cost. However, there are some limitations in our study. Firstly, the sample size is relatively small, further studies are required to confirm the application of this modified technique in the future; Secondly, this modification lacks the partial bicortical purchase, and this difference may account for the reduced TLFS stiffness in axial rotation and lateral bending because the screws may toggle within the cancellous laminar bone. Therefore, the question of whether this technique is biomechanically as effective as the traditional TLFS method is still open to further research. Finally, although, compare with conventional TLIF, our modified technique seems to be more minimal invasive, a better comparison to other minimal invasive fixation (such as MIS-TLIF, in which no contralateral subperiosteal dissection is needed cause contralateral screws replaced percutaneously.) may lead to more clinical significance, which warrant further studies.

\section{Conclusions}

In our modified technique, a tiny unicortical "hole" was created at the dorsal lamina adjacent to the contralateral facet joint. By allowing direct visualization of the insertion of the screw against the dorsal cortices of the lamina, the modified technique can reduce soft tissue injuries, reduce the risk of violating the spinal canal, minimize radiation exposure, and shorten the operation time without a concurrent reduction in clinical efficacy, in contrast to conventional TLIF.

\section{Abbreviations}


TLFS: Translaminar facet screws; TLIF: Transforaminal lumbar interbody fusion; BPS: Bilateral pedicle screws; UPS: Unilateral pedicle screw; CT: Computed tomography; AP: Anteroposterior.

\section{Declarations}

\section{Ethics approval and consent to participate}

This study was approved by the ethics committee of the Second Affiliated Hospital and Yuying Children's Hospital of Wenzhou Medical University. The approval number is L-2016-09. As for this research, an optout of the informed consent, the information disclosure, and a negative opportunity are guaranteed in the Ethical approval.

\section{Consent for publication}

All the patients in this study have given their informed consent for the article to be published.

\section{Availability of data and materials}

The datasets used and/or analyzed during the current study are available from the corresponding author on reasonable request.

\section{Competing interests}

The authors declare that they have no competing interests.

\section{Funding}

No funding was provided specifically for this study.

\section{Authors' contributions}

All authors have made substantial contributions to the conception and design, acquisition of data, and analysis and interpretation. All authors have been involved in drafting the manuscript or revising it critically for important intellectual content. All authors have given final approval of the version to be published. All authors agree to be accountable for all aspects of the work in ensuring that questions related to the accuracy or integrity of any part of the work are appropriately investigated and resolved.

\section{Acknowledgements}

Not applicable.

\section{References}

1. Harms J, Rolinger H. [A one-stager procedure in operative treatment of spondylolistheses: dorsal traction-reposition and anterior fusion (author's transl)]. Zeitschrift fur Orthopadie ihre Grenzgebiete. 1982;120:343-7. doi:10.1055/s-2008-1051624.

2. Lowe TG, Tahernia AD, O'Brien MF, Smith DA. Unilateral transforaminal posterior lumbar interbody fusion (TLIF): indications, technique, and 2-year results. J Spin Disord Tech. 2002;15:31-8. 
3. Potter BK, Freedman BA, Verwiebe EG, Hall JM, Polly DW Jr, Kuklo TR. Transforaminal lumbar interbody fusion: clinical and radiographic results and complications in 100 consecutive patients. $J$ Spin Disord Tech. 2005;18:337-46.

4. Suk KS, Lee HM, Kim NH, Ha JW. Unilateral versus bilateral pedicle screw fixation in lumbar spinal fusion. Spine. 2000;25:1843-7. doi:10.1097/00007632-200007150-00017.

5. Slucky AV, Brodke DS, Bachus KN, Droge JA, Braun JT. Less invasive posterior fixation method following transforaminal lumbar interbody fusion: a biomechanical analysis. The spine journal: official journal of the North American Spine Society. 2006;6:78-85. doi:10.1016/j.spinee.2005.08.003.

6. Jang JS, Lee SH. Minimally invasive transforaminal lumbar interbody fusion with ipsilateral pedicle screw and contralateral facet screw fixation. Journal of neurosurgery Spine. 2005;3:218-23. doi:10.3171/spi.2005.3.3.0218.

7. Awad BI, Lubelski D, Shin JH, Carmody MA, Hoh DJ, Mroz TE, Steinmetz MP. Bilateral Pedicle Screw Fixation versus Unilateral Pedicle and Contralateral Facet Screws for Minimally Invasive Transforaminal Lumbar Interbody Fusion: Clinical Outcomes and Cost Analysis. Global spine journal. 2013;3:225-30. doi:10.1055/s-0033-1349399.

8. Cao Y, Chen Z, Jiang C, Wan S, Jiang X, Feng Z. The combined use of unilateral pedicle screw and contralateral facet joint screw fixation in transforaminal lumbar interbody fusion. European spine journal: official publication of the European Spine Society, the European Spinal Deformity Society, and the European Section of the Cervical. Spine Research Society. 2015;24:2607-13. doi:10.1007/s00586-015-4120-x.

9. Sethi A, Muzumdar AM, Ingalhalikar A, Vaidya R. Biomechanical analysis of a novel posterior construct in a transforaminal lumbar interbody fusion model an in vitro study. The spine journal: official journal of the North American Spine Society. 2011;11:863-9. doi:10.1016/j.spinee.2011.06.015.

10. Grob D, Humke T. Translaminar screw fixation in the lumbar spine: technique, indications, results. European spine journal: official publication of the European Spine Society, the European Spinal Deformity Society, and the European Section of the Cervical. Spine Research Society. 1998;7:178-86. doi:10.1007/s005860050053.

11. Humke T, Grob D, Dvorak J, Messikommer A. Translaminar screw fixation of the lumbar and lumbosacral spine. A 5-year follow-up. Spine. 1998;23:1180-4. doi:10.1097/00007632-19980515000021.

12. Zeng ZY, Zhang JQ, Song YX, Yan WF, Wu P, Tang HC, Han JF. Combination of percutaneous unilateral translaminar facet screw fixation and interbody fusion for treatment of lower lumbar vertebra diseases: a follow-up study. Orthopaedic surgery. 2014;6:110-7. doi:10.1111/os.12100.

13. Lu J, Ebraheim NA, Yeasting RA. Translaminar facet screw placement: an anatomic study. American journal of orthopedics (Belle Mead NJ). 1998;27:550-5. 
14. Lauber S, Schulte TL, Liljenqvist U, Halm H, Hackenberg L. Clinical and radiologic 2-4-year results of transforaminal lumbar interbody fusion in degenerative and isthmic spondylolisthesis grades 1 and 2. Spine. 2006;31:1693-8. doi:10.1097/01.brs.0000224530.08481.4e.

15. Tuli J, Tuli S, Eichler ME, Woodard EJ. A comparison of long-term outcomes of translaminar facet screw fixation and pedicle screw fixation: a prospective study. Journal of neurosurgery Spine. 2007;7:287-92. doi:10.3171/spi-07/09/287.

16. Kandziora F, Schleicher P, Scholz M, Pflugmacher R, Eindorf T, Haas NP, Pavlov PW. Biomechanical testing of the lumbar facet interference screw. Spine. 2005;30:E34-9. doi:10.1097/01.brs.0000150484.85822.d0.

17. Kim SM, Lim TJ, Paterno J, Kim DH. A biomechanical comparison of supplementary posterior translaminar facet and transfacetopedicular screw fixation after anterior lumbar interbody fusion. Journal of neurosurgery Spine. 2004;1:101-7. doi:10.3171/spi.2004.1.1.0101.

18. Schleicher P, Beth P, Ottenbacher A, Pflugmacher R, Scholz M, Schnake KJ, Haas NP, Kandziora F. Biomechanical evaluation of different asymmetrical posterior stabilization methods for minimally invasive transforaminal lumbar interbody fusion. Journal of neurosurgery Spine. 2008;9:363-71. doi:10.3171/spi.2008.9.10.363.

19. Magerl FP. (1984) Stabilization of the lower thoracic and lumbar spine with external skeletal fixation. Clinical orthopaedics and related research:125-141.

20. Jacobs RR, Montesano PX, Jackson RP. Enhancement of lumbar spine fusion by use of translaminar facet joint screws. Spine. 1989;14:12-5. doi:10.1097/00007632-198901000-00003.

21. Montesano PX, Magerl F, Jacobs RR, Jackson RP, Rauschning W. Translaminar facet joint screws. Orthopedics. 1988;11:1393-7.

22. Jang JS, Lee SH, Lim SR. Guide device for percutaneous placement of translaminar facet screws after anterior lumbar interbody fusion. Technical note. Journal of neurosurgery. 2003;98:100-3. doi:10.3171/spi.2003.98.1.0100.

23. $10.1097 / 01$. brs.0000157473.17313.6f

Shim CS, Lee SH, Jung B, Sivasabaapathi P, Park SH, Shin SW. (2005) Fluoroscopically assisted percutaneous translaminar facet screw fixation following anterior lumbar interbody fusion: technical report. Spine 30:838-843. doi: 10.1097/01.brs.0000157473.17313.6f.

24. Amoretti N, Amoretti ME, Hovorka I, Hauger O, Boileau P, Huwart L. Percutaneous facet screw fixation of lumbar spine with CT and fluoroscopic guidance: a feasibility study. Radiology. 2013;268:548-55. doi:10.1148/radiol.13120907.

25. Kang HY, Lee SH, Jeon SH, Shin SW. Computed tomography-guided percutaneous facet screw fixation in the lumbar spine. Technical note. Journal of neurosurgery Spine. 2007;7:95-8. doi:10.3171/spi-07/07/095.

26. Phillips FM, Ho E, Cunningham BW. Radiographic criteria for placement of translaminar facet screws. The spine journal: official journal of the North American Spine Society. 2004;4:465-7. 


\section{Figures}
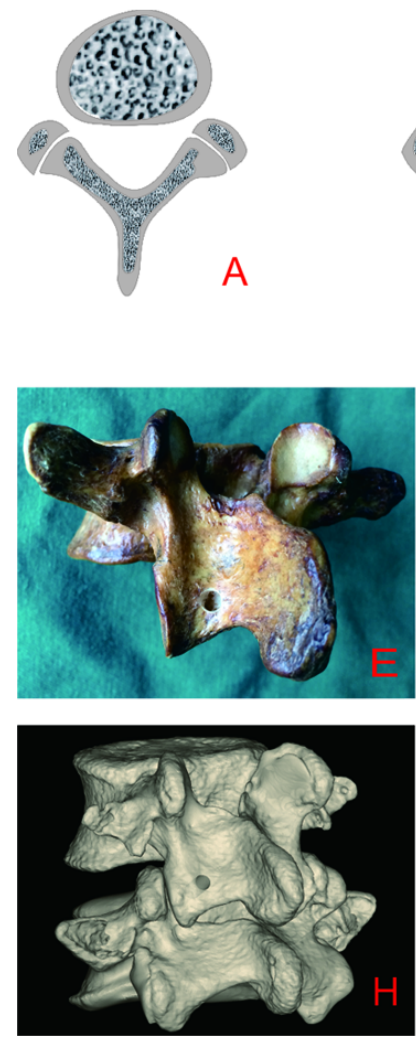
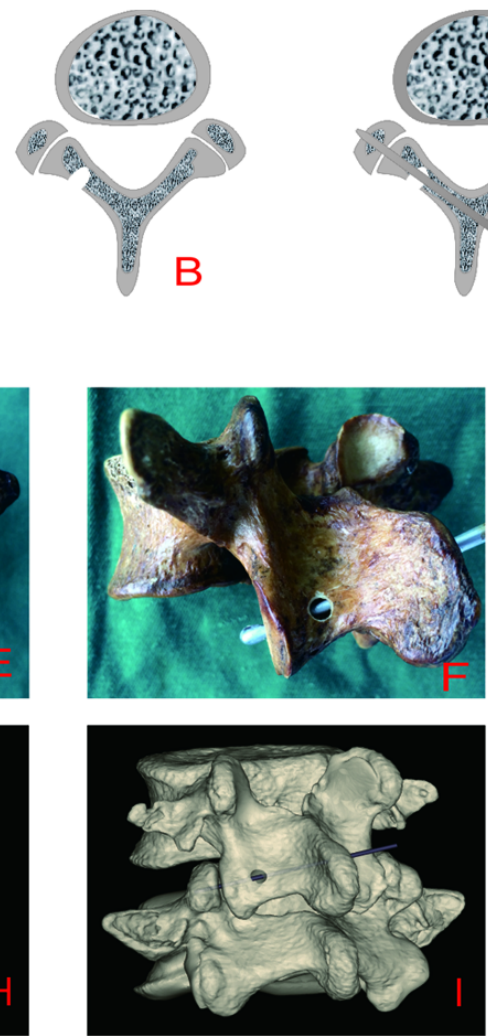
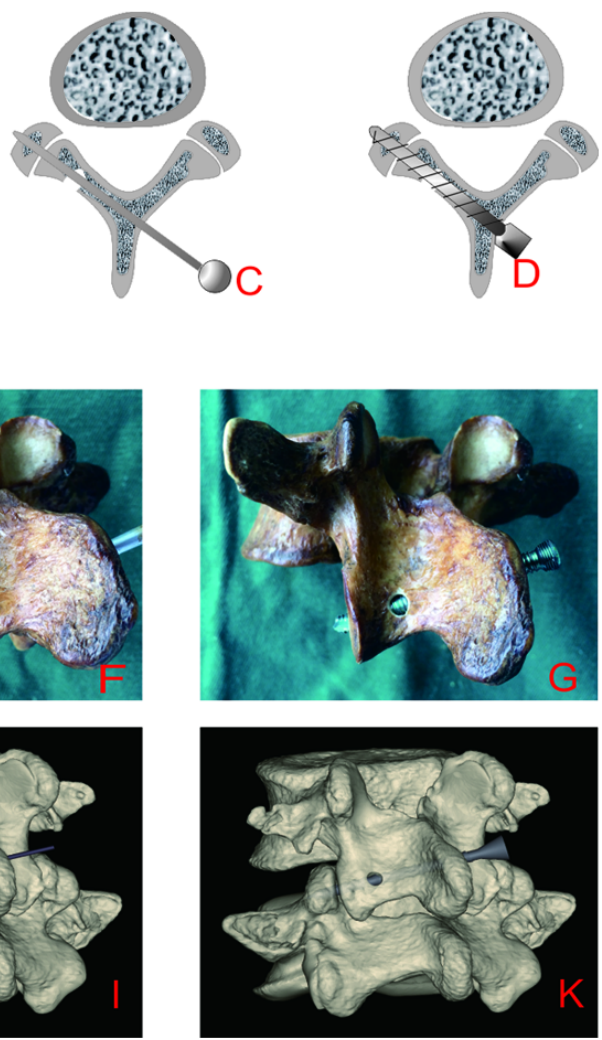

Figure 1.

(A) Superior view of the lumbar vertebral body. A small unicortical "hole" adjacent to the facet joint at contralateral lamina was made by a diamond dri11. (B, E, H)

With the drill visually aligned along the angle of the exposed contralateral laminar surface, the lamina was carefully drilled along its length, aiming for the unicortical "hole" adjacent to the contralateral facet joint. (C, F, I) The screw can be directly visualized through the unicortical “hole”. (D, G, K)

\section{Figure 1}

(A) Superior view of the lumbar vertebral body. $(B, E, H)$ A small unicortical "hole" adjacent to the facet joint at contralateral lamina was made by a diamond drill. $(C, F, I)$ With the drill visually aligned along the angle of the exposed contralateral laminar surface, the lamina was carefully drilled along its length, aiming for the unicortical "hole" adjacent to the contralateral facet joint. (D, G, K) The screw can be directly visualized through the unicortical "hole". 

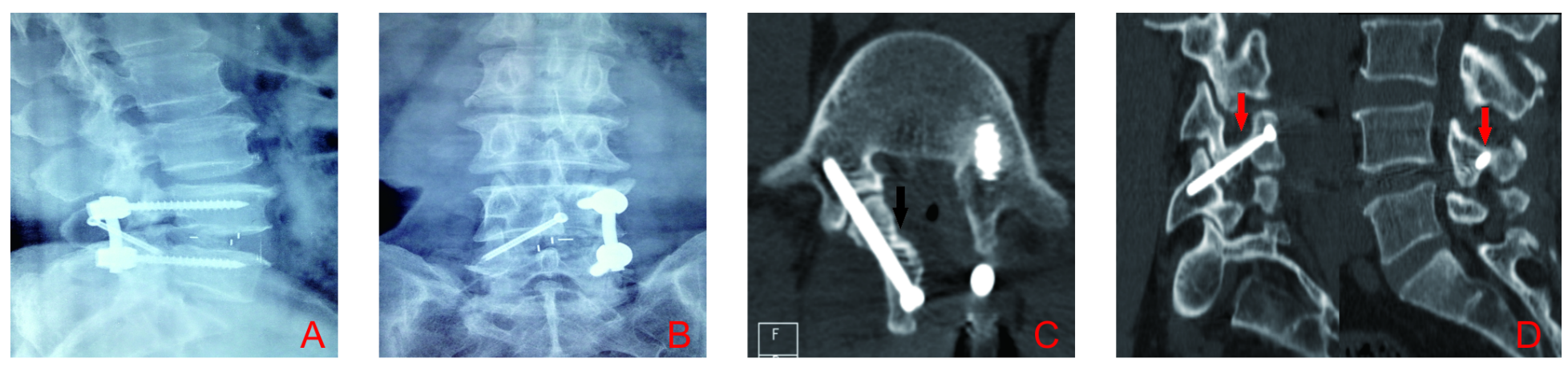

Figure 2. Postoperative plain lumbar spine $\mathrm{X}$-ray views showed the TLIF with UPS+TLFS. (A, B) Postoperative axial and sagittal reconstructed CT scans showed that the position of the screw was satisfactory and no violating of the spinal canal. (C, D) (black arrow in $\mathrm{C}$ and red arrows in D)

\section{Figure 2}

(A, B) Postoperative plain lumbar spine X-ray views showed the TLIF with UPS+TLFS. (C, D) Postoperative axial and sagittal reconstructed CT scans showed that the position of the screw was satisfactory and no violating of the spinal canal (black arrow in $\mathrm{C}$ and red arrows in $\mathrm{D}$ ) 